\title{
IN-SITU DYNAMIC FORCE CALIBRATION USING IMPACT HAMMERS
}

\author{
M. Kobusch ${ }^{1}$, L. Klaus ${ }^{2}$ \\ Physikalisch-Technische Bundesanstalt (PTB), Bundesallee 100, 38116 Braunschweig, Germany, \\ ${ }^{1}$ michael.kobusch@ptb.de, ${ }^{2}$ leonard.klaus@ptb.de
}

\begin{abstract}
:
This paper presents experimental investigations of in-situ dynamic force calibrations in which an impact hammer provides the dynamic reference force. Here, the force transducer to be calibrated remains in the original mechanical structure of the force measurement application to which calibration shock forces are applied in a suitable way. Numerous experiments with different force transducer set-ups and different impact hammer configurations were conducted to validate this insitu calibration method. The paper describes the analysis of the measurement data and presents the force transfer functions obtained. Finally, these dynamic calibration results are discussed.
\end{abstract}

Keywords: shock force; dynamic calibration; transfer function; modal hammer

\section{INTRODUCTION}

While the measurement of dynamic forces plays an important role in many areas of industry, the traceability of such forces is predominantly based on static calibrations, as documentary standards and generally accepted guidelines for dynamic calibrations rarely exist or are still in their infancy $[1,2]$.

Research on the dynamic calibration of force transducers has been conducted by several national metrology institutes in recent years. The main objective of this research is the establishment of calibration facilities in which sinusoidal or pulseshaped force excitations are used. Although these facilities are suited to characterize a force transducer dynamically, the calibration results obtained by means of different excitation methods or calibration set-ups can often only be transferred to each other, or later to a specific application, via suitable mechanical modelling [3]. For this purpose, corresponding decisive dynamic parameters must be determined that result from the given mass and elasticity distribution of the force transducer and the calibration set-up connected to it.

To avoid the difficulties and challenges of dynamic force traceability associated with a transducer dynamically calibrated in an external device, researchers from the American national metrology institute NIST proposed in-situ calibration by means of a dynamically calibrated impact hammer used as a force reference [4]. Furthermore, an application of a dynamically uncalibrated impact hammer to characterize the dynamic behaviour of a force transducer array was published in [5].

Impact hammers (or modal hammers) are widely used in industry and science for the modal testing of mechanical structures. These hammers incorporate a piezoelectric force sensor that measures the force pulses applied. Interchangeable hammer tips and mass extenders are used to vary the length and amplitude, and thus the spectral content, of the pulses. Further information on the theory and practice of dynamic measurements and modal analysis by means of impact hammers can be found in $[6,7]$.

Such an in-situ calibration has a significant advantage: the force transducer to be calibrated remains unchanged in its original mechanical set-up as used for the dynamic measurement application. Possible changes in the dynamic system behaviour resulting from mounting and dismounting the force transducer (e.g. due to variations of contact stiffness, damping, component positioning or orientation) are avoided. In addition, no complex modelling or compensation of parasitic inertial force components are required.

The in-situ calibration should be performed using the same amplifier and data acquisition system, configurations, filter settings and sampling rates that will be used later for dynamic force measurements. Otherwise, the frequency responses of the different measuring chains must be known and taken into account accordingly. The in-situ dynamic force calibration provides calibration results in the frequency domain - the frequency response function which, in principle, allows traceability to other dynamic signal forms such as sine forces, step forces or arbitrary force pulses.

In the following, experimental tests of the in-situ dynamic calibration method proposed are presented in which the measurement set-up uses a similar mechanical arrangement of a force transducer 
mounted at its base and loaded at its top by a load mass to which the shock force is applied. The use of different impact hammers as well as different measurement set-ups is simulated by varying the hammer configuration and changing the load mass of the transducer, respectively. This mechanical configuration is typical for many impact force measurement applications in industry such as component tests and crash tests.

\section{HAMMER CALIBRATION}

The in-situ dynamic force calibration method proposed is a secondary calibration method in which the reference force is provided by a dynamically calibrated impact hammer. In contrast to the negative force step calibration method used in [4], calibrations at PTB are carried out on a conventional hammer calibration set-up that provides traceability by measuring the inertial force of a reference mass that has been hit by the impact hammer to be calibrated. The calibration device shown in Figure 1 uses a string-suspended cylindrical mass body (e.g. $1 \mathrm{~kg}$ ) that is struck by the hammer. The inertial force of this mass body is measured by an accelerometer and is compared to the hammer's output signal. In order to simulate the curved movement of manually executed hammer strokes and to achieve a defined impact trajectory that can be reproduced well, the hammer is elastically clamped in a holder mounted on a pendulum.

All measurements described in this paper were carried out with a Kistler 9726A200001 impact hammer connected to an IEPE signal conditioner (PCB 482C series) that forms part of the measuring chain considered. The hammer that is specified with a head mass of $500 \mathrm{~g}$ was equipped with different hammer tips and an optional mass extender $(250 \mathrm{~g})$ screwed onto the hammer head. All components of the force reference (acceleration sensor, charge amplifier, reference mass) were calibrated and traced to SI units. Both calibration signals were sampled by means of a National Instruments PXI-5922 data acquisition card.

Whereas the calibration result commonly used in the time domain is the sensitivity defined as the pulse peak ratio, this paper will focus on calibration results in the frequency domain, i.e. the complex frequency response (sensitivity) in magnitude and phase. However, only magnitude responses are presented in the following, as these have the most practical importance in dynamic force measurement.

Typical pulse signals of the impact hammer calibration are visualized in Figure 2 for two different hammer tips. All signals were passed through a 4th order $20 \mathrm{kHz}$ Bessel low-pass filter to suppress disturbances caused by modal vibrations of the acceleration sensor or the reference mass observed above $50 \mathrm{kHz}$. The acceleration signal obtained with the steel tip still shows some remaining ripples. The pulse width weakly depends on the pulse magnitude [8] due to the nonlinear contact stiffness of the hammer tip and is about $0.2 \mathrm{~ms}$ (steel tip) or $0.65 \mathrm{~ms}$ (plastic tip) using a reference mass of $1 \mathrm{~kg}$. Both calibration signals show very similar Gaussian-like pulse shapes.

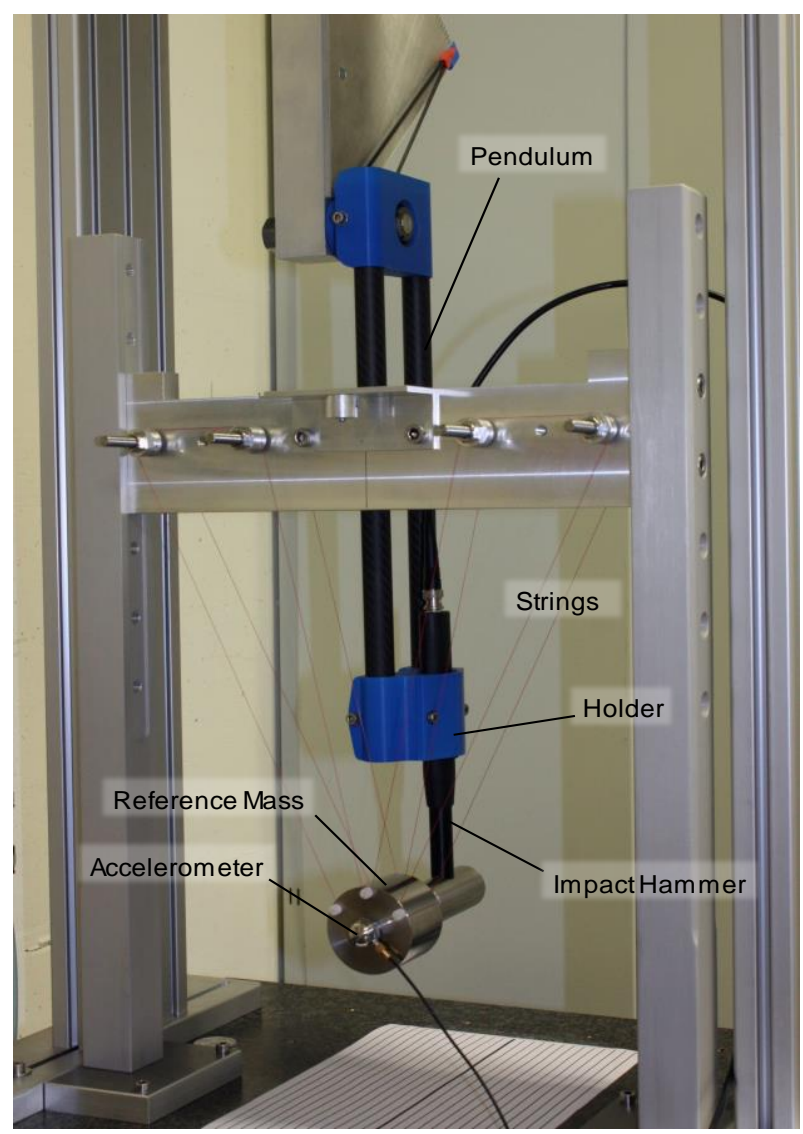

Figure 1: Impact hammer calibration device at PTB

The magnitude response of four impact hammer configurations - applying two different hammer tips with and without a mass extender - is visualized in Figure 3; diagram (a) shows the response in the lower frequency range up to a frequency of $2 \mathrm{kHz}$, (b) up to $10 \mathrm{kHz}$. Each curve represents the weighted spectral average of pulses with different shock force magnitudes and is given with its respective weighted standard deviation. The weighting is based on the power spectral density of the reference signal. The number of samples ranges from 12 to 33 and is indicated in brackets. At a sample length of $15 \mathrm{~ms}$, the frequency resolution of the discrete Fourier transform (DFT) amounts to $67 \mathrm{~Hz}$.

\footnotetext{
${ }^{1}$ Commercial devices are identified in this paper only to adequately specify the experimental set-up. Such identification does not imply recommendation by PTB, nor does it imply that the equipment identified is necessarily the best available for the purpose.
} 
The magnitude responses are almost constant at lower frequencies and the four configurations differ by less than $2.5 \%$ up to a frequency of $2 \mathrm{kHz}$. At higher frequencies, the curves clearly decrease, and measurements with the plastic tip demonstrate apparent ripples of unknown origin. In general, hammer calibrations with the metal tip or the mass extender yield slightly higher values.

Above $2 \mathrm{kHz}$, the weighted standard deviation strongly increases for measurements with the plastic tip, which indicates an insufficient frequency content for large pulse widths. The increased deviations at the lowest frequency bins are due to power line noise.

The coherence function is commonly used as a measure of the correlation between two signals in the frequency domain. Figure 4 shows the coherence of the measurements presented. The values decrease substantially at higher frequencies, especially when measuring with the plastic tip. In this case, the usable frequency range of the hammer calibration data is limited to a few kilohertz, whereas the steel tip can be used up to $10 \mathrm{kHz}$.
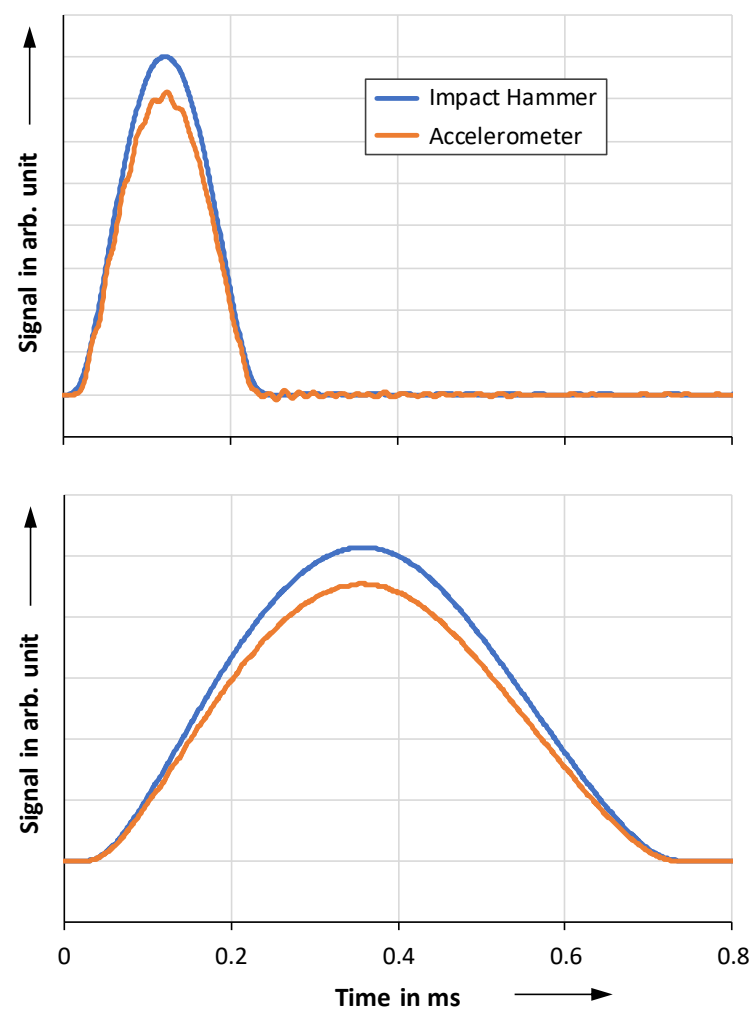

Figure 2: Calibration signals of impact hammer (blue) and acceleration sensor (orange) obtained with hammer tips made of steel (top) and plastic (bottom).

In order to estimate the measurement uncertainties of the impact hammer calibration, further research is needed to understand the observed behaviour. Possible influences are elastic modes of the hammer or the reference mass. However, the lowest modal resonance of the $1 \mathrm{~kg}$ reference mass is at about $30 \mathrm{kHz}$ [8].
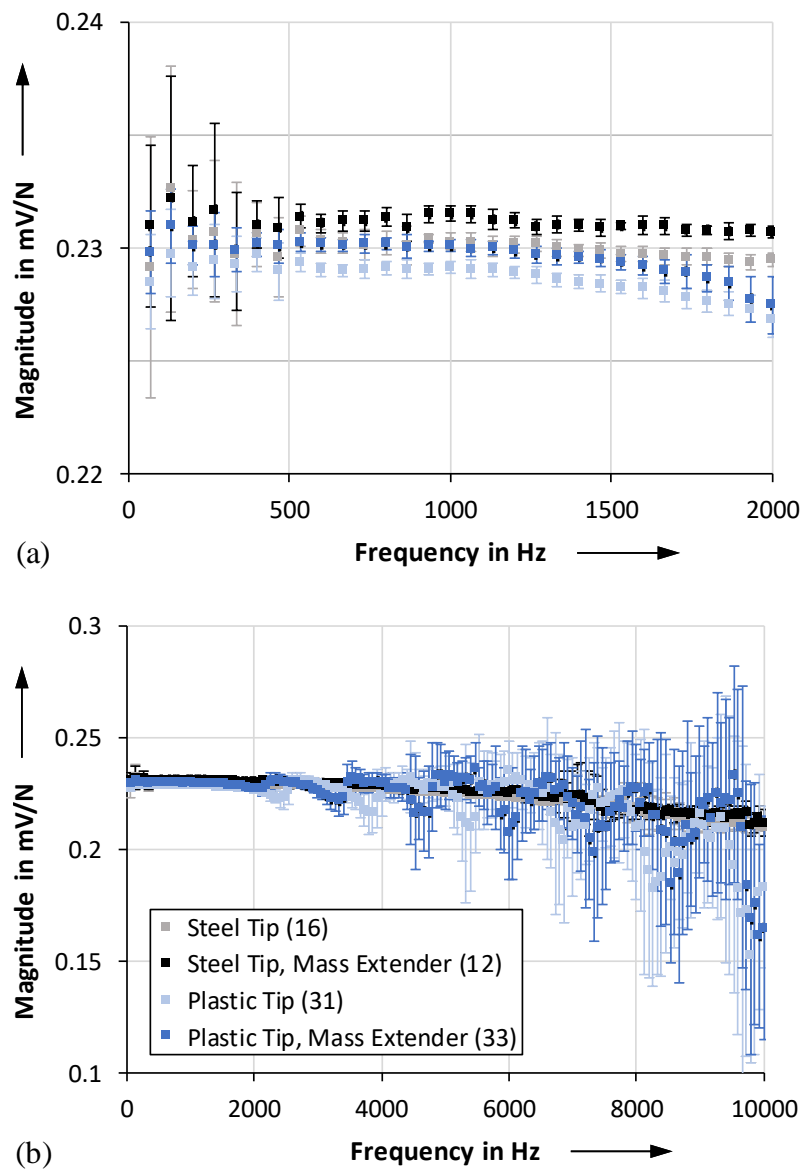

Figure 3: Magnitude response of the impact hammer for four hammer configurations: (a) frequency range up to $2 \mathrm{kHz}$, (b) up to $10 \mathrm{kHz}$. Each curve shows the weighted mean with the weighted standard deviation. The number of samples is given in brackets.

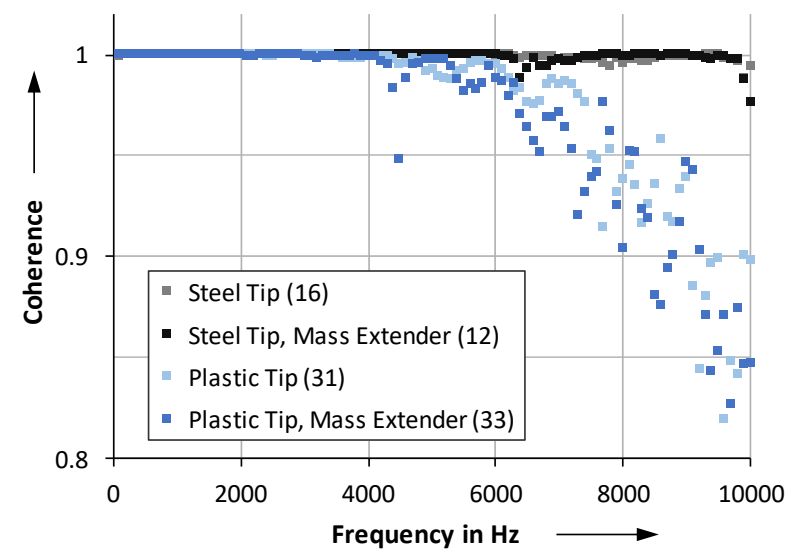

Figure 4: Coherence between the two calibration signals for four hammer configurations; each curve shows the arithmetic mean. The sample size is given in brackets.

The rather flat sensitivity of the tested impact hammer seems to validate its use as a dynamic force reference in the lower kilohertz range. Even if only the static calibration or a single-frequency calibration values were used, the potential dynamic errors would be quite small for many dynamic applications. In the following section, the suitability as a dynamic force standard for in-situ calibrations is experimentally investigated. 


\section{IN-SITU DYNAMIC FORCE CALIBRATION EXPERIMENTS}

The principal arrangement of the in-situ dynamic force calibration set-up is illustrated in Figure 5. The force transducer to be calibrated (signal $U_{\mathrm{FT}}(t)$ ) and the reference force sensor (signal $U_{\mathrm{REF}}(t)$ ) incorporated in the impact hammer are marked in blue. The force transducer is vertically mounted on a heavy base plate by means of an adapter; its upper section consists of a load adapter and an optional load mass fixed in place by screws. The hammer is operated by hand in such a way that the hammer tip hits the load adapter as centrally and vertically as possible. Photographs of the respective mechanical components of the experiments are shown in Figures 6 and 7.

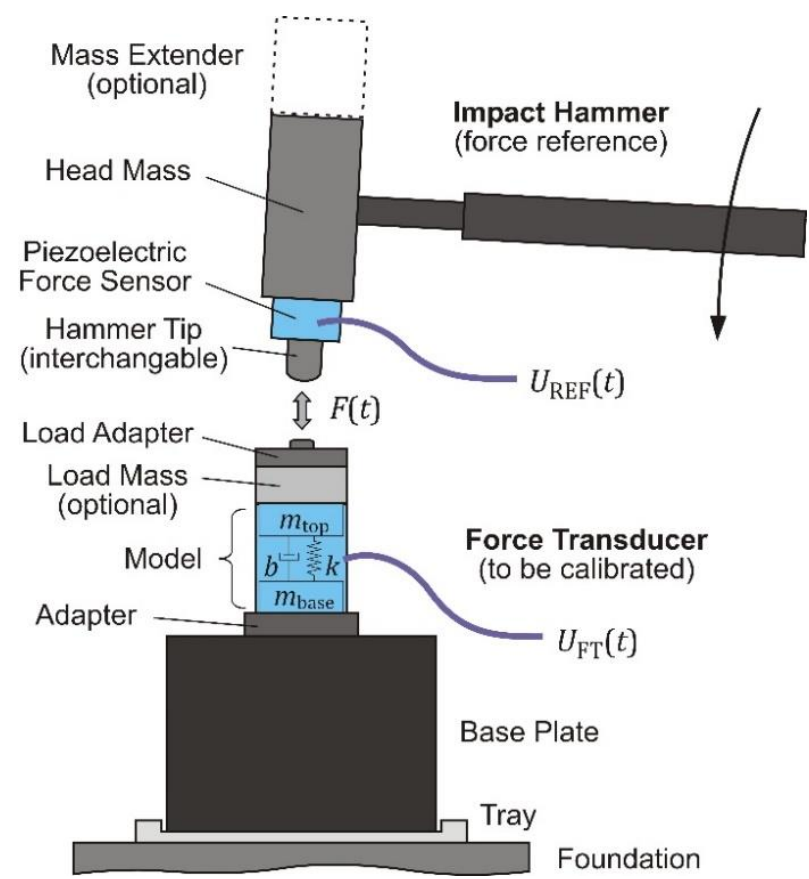

Figure 5: Illustration of the in-situ dynamic force calibration.

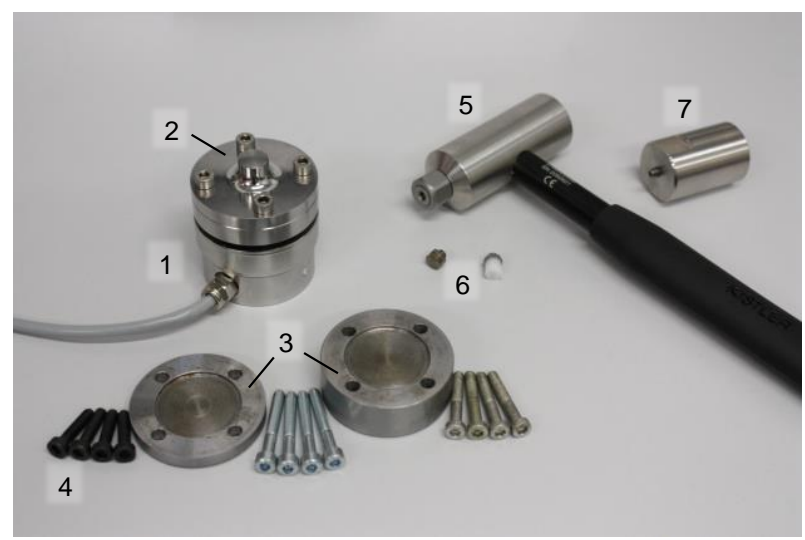

Figure 6: Components of the calibration experiments: HBM U3/10 kN force transducer (1) with load adapter (2) and two optional load mass discs (3) plus mounting screws (4); Kistler 9726A20000 impact hammer (5) with two hammer tips (6) and optional mass extender (7).
The in-situ dynamic calibration set-up can be modelled as a mass-spring-damper system; in the simplest approach with one degree of freedom, it is attached to a rigid base and its mass is struck by the hammer. The model mass consists of the applied load components plus the internal upper part of the force transducer, and the spring represents the transducer's axial stiffness. The larger the mass, the lower the system's fundamental resonant frequency.

The tests were carried out using a small strain gauge force transducer (HBM U3 / $10 \mathrm{kN})$ mounted to a steel mass cube of $10 \mathrm{~kg}$ as the base plate, which rested freely on the foundation below via a plastic tray. This mass is the reaction mass of the $20 \mathrm{kN}$ impact force calibration facility at PTB, thus simplifying future comparison calibrations. The force transducer is equipped with flange ends on both sides and features an integrated lateral force compensation, which is advisable for manual hammer blows where the impact position and force direction are not well defined. The hammer force was introduced to the upper flange end of the force transducer via a screwed-on load adapter with a spherical load button. To simulate mechanically different in-situ dynamic calibration set-ups, the top end of the force transducer was equipped with different loads. Stacking the load adapter with two optional mass discs plus mounting bolts yielded four values determined by weighing: $135 \mathrm{~g}, 237 \mathrm{~g}$, $446 \mathrm{~g}$ and $551 \mathrm{~g}$.

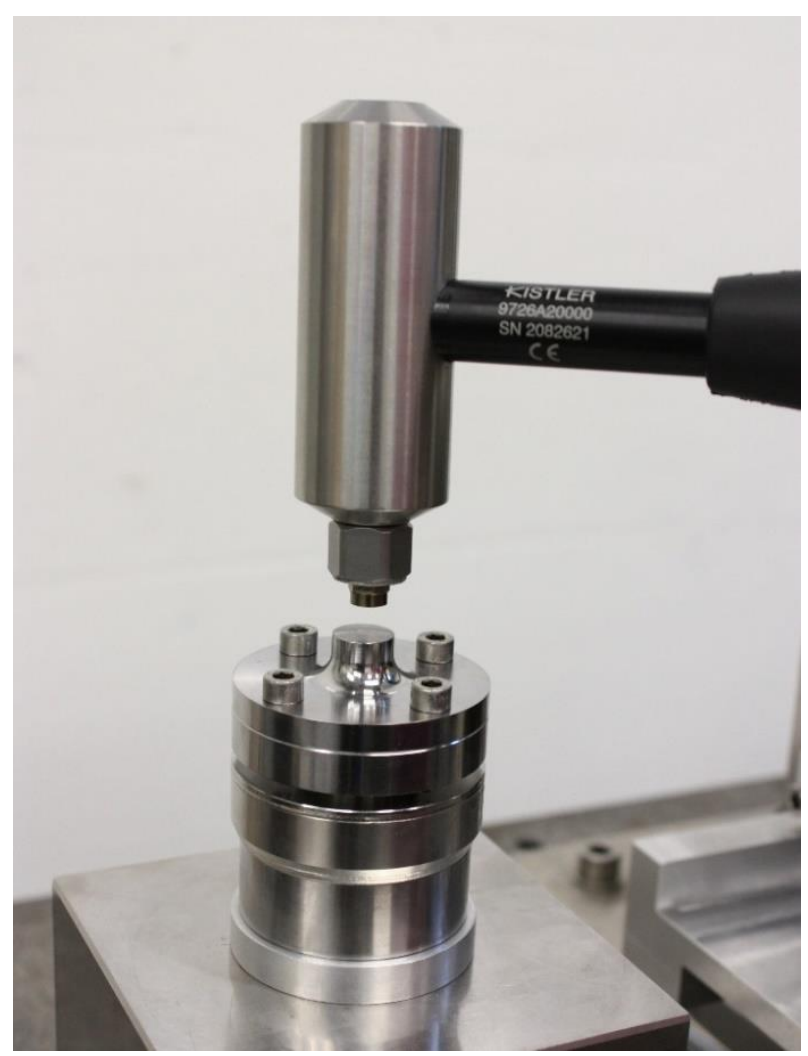

Figure 7: In-situ dynamic force calibration using a load mass of $135 \mathrm{~g}$ and a steel hammer tip. 
All tests were performed with a single impact hammer; however, in a practical sense, the use of different hammer tips and an optional mass extension simulated the application of different hammers. For each combination of a force transducer set-up and a hammer configuration, hammer strokes of varying force magnitude were measured and analysed.

Figure 8 presents typical pulse signals in the time domain for four experimental configurations. The upper row was obtained with $135 \mathrm{~g}$ (the smallest external load mass), the bottom row with $446 \mathrm{~g}$. The signals achieved with the steel hammer tip are shown on the left and those achieved with the plastic tip are shown on the right. In each example, the hammer peak force was in the range of $1.5 \mathrm{kN}$.
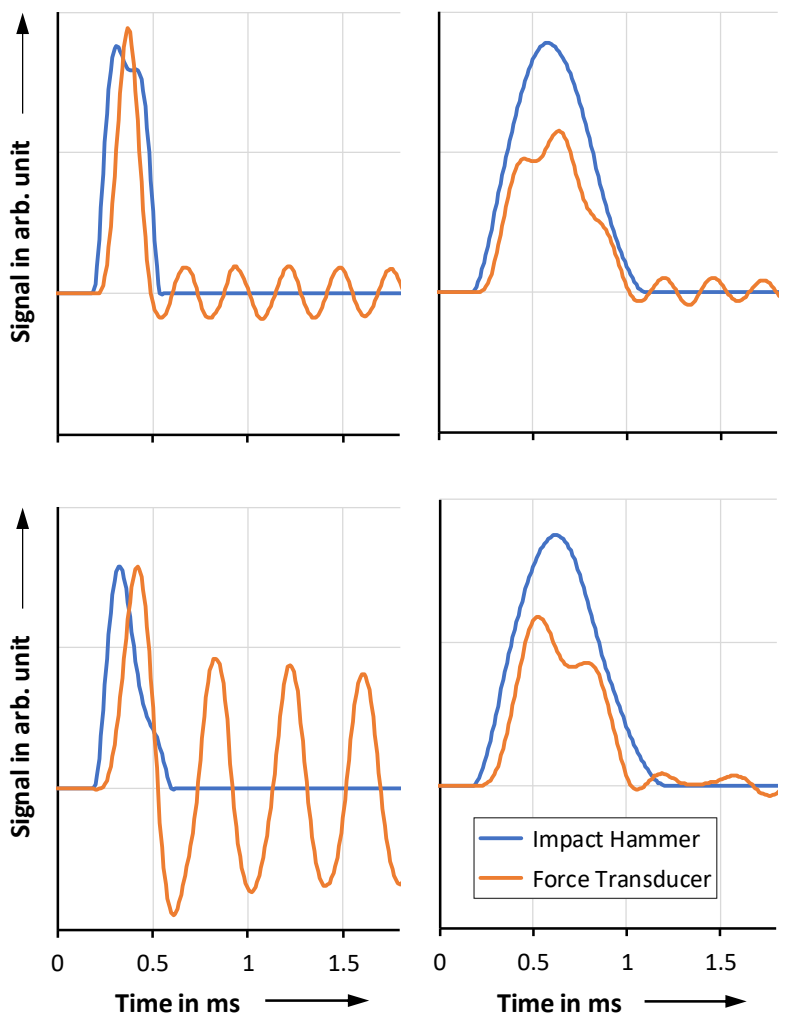

Figure 8: Measurement signals of the in-situ dynamic force calibration for four experimental configurations: HBM U3/10 kN force transducer with a load of $135 \mathrm{~g}$ (top row) and $446 \mathrm{~g}$ (bottom row); hammer strokes with steel tip (left) and plastic tip (right).

These examples clearly demonstrate that the pulse shape and the peak force of the impact hammer and the force transducer can deviate substantially, i.e. the comparison of peak values is not useful. The fundamental resonance is excited by the hammer strokes and the force transducer measures a ringing whose vibration amplitude depends on the specific configuration and impact conditions.

The following diagrams present the magnitude response for different configurations of the calibration set-up and the impact hammer. Each curve displays averaged data from about 40 measurements. The pulse peak values of the impact hammer ranged from about $0.3 \mathrm{kN}$ to $2.5 \mathrm{kN}$.

Figure 9 demonstrates the effect of the variation of the load mass on the magnitude response showing measurements obtained with the steel hammer tip. The upper diagram (a) visualizes the data up to a frequency of $4 \mathrm{kHz}$ on a logarithmic axis scale, the magnified view (b) up to $2 \mathrm{kHz}$ on a linear scale.

The three curves start at similar magnitudes - the force transducer is specified with a static sensitivity of $0.5 \mathrm{mV} / \mathrm{N}$ - and gradually rise to a dominant resonance, whose frequency strongly depends on the applied load mass. This resonance is described by the above-mentioned model with one degree of freedom. The magnified view shows that the magnitude responses also differ at lower frequencies. The kink at the lowest frequency bins is caused by the rather elastic coupling of the $10 \mathrm{~kg}$ base plate with the calibration set-up to the foundation below. This dynamic behaviour was confirmed by modelling the magnitude response function using a multi-degrees-of-freedom model that considered an elastic base coupling of the calibration set-up.
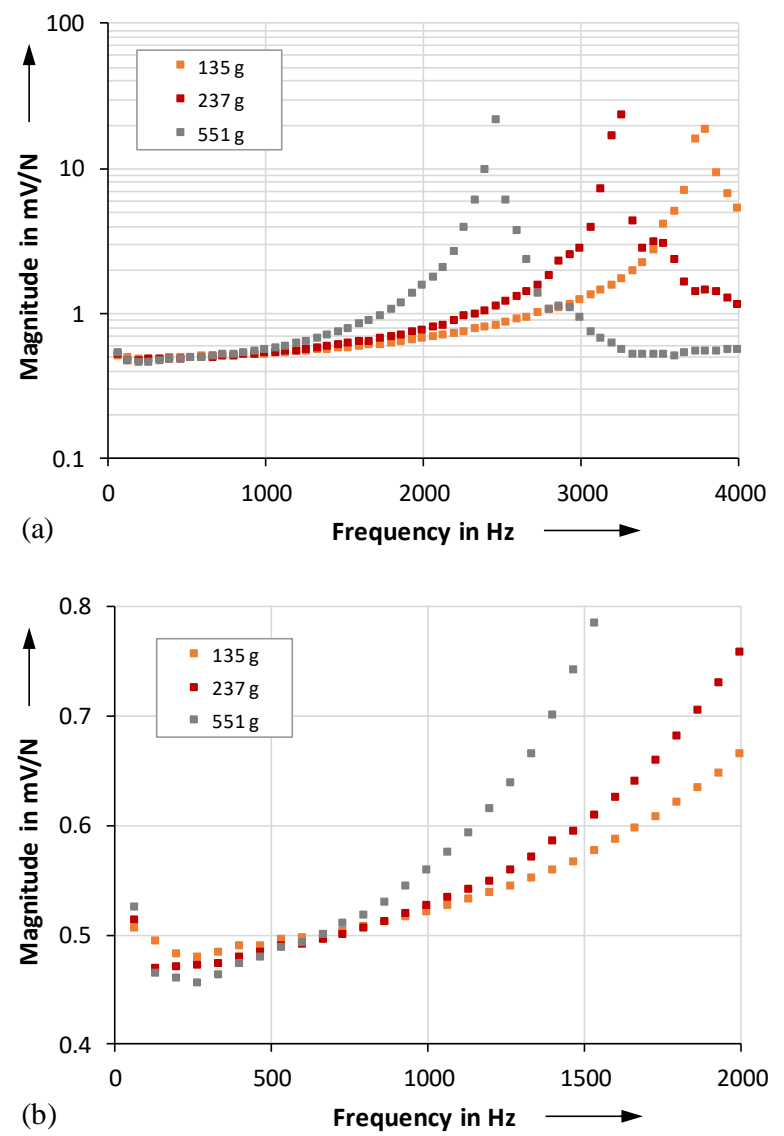

Figure 9: Magnitude response for three different force calibration set-ups: load masses $135 \mathrm{~g}, 237 \mathrm{~g}, 551 \mathrm{~g}$; impact hammer with steel tip; (a) resonance peaks in the frequency range up to $4 \mathrm{kHz}$; (b) magnified view of the lower frequency range up to $2 \mathrm{kHz}$.

The influence of the impact hammer on the magnitude response of the calibration set-up is shown in Figure 10 for an example load mass of 
$237 \mathrm{~g}$. The measurements were obtained with both hammer tips, both with and without a mass extender. As can be seen, the four responses agree very well.

The agreement of calibration results obtained with different hammer configurations and experimentally tested on different calibration setups allows the conclusion that the in-situ calibration method proposed is well suited for dynamic force calibrations, at least for mechanical arrangements similar to those investigated in this work.

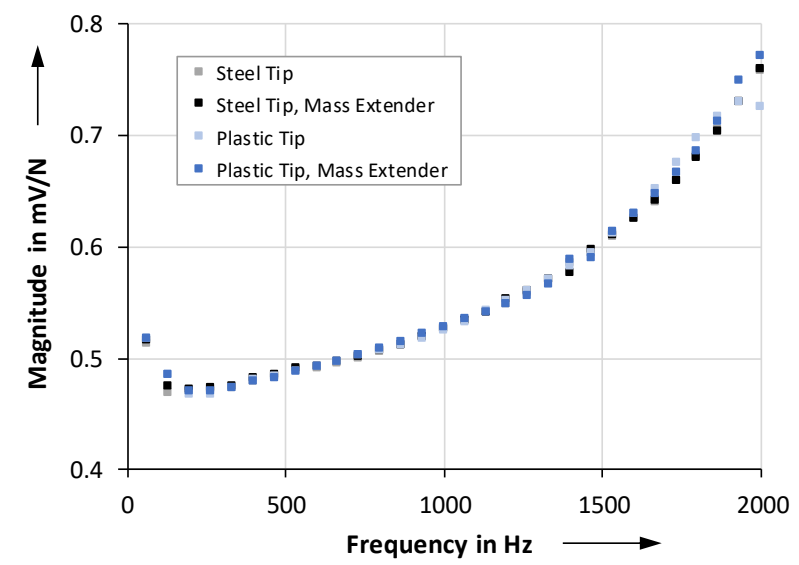

Figure 10: Magnitude response for different hammer configurations: steel/plastic hammer tip; with/without mass extender; load mass $237 \mathrm{~g}$.

\section{SUMMARY AND OUTLOOK}

This paper presents experimental investigations of a dynamic in-situ force calibration method recently proposed by NIST. This secondary calibration method uses a dynamically calibrated impact hammer to provide the dynamic reference force. A dynamic in-situ calibration has a considerable advantage: the force transducer to be calibrated remains in its original mechanical structure for the subsequent dynamic force measurement; furthermore, the many difficulties and challenges associated with an external dynamic calibration can be avoided. The in-situ calibration measurements are preferably performed with the same measuring electronics and the same settings as are later used for dynamic force measurements, so that further corrections do not have to be made.

Although all experiments presented in this paper were carried out using a single force transducer and a single calibration hammer, numerous variations of the mechanical structures of the calibration set-up and the force generation allow a generalised statement on the usability of the calibration method investigated. Experiments on different calibration set-ups prove that calibration results obtained with different hammer configurations agree well; it can thus be concluded that impact hammers are suited to provide a dynamic in-situ force calibration. The calibration results expressed in the frequency domain will in principle provide traceability to any dynamic signal shape within the considered bandwidth; i.e. the in-situ calibration by means of an impact hammer is not restricted to shock force applications.

Future work will focus on the estimation of the measurement uncertainties of the hammer calibration as well as the in-situ force calibration. In this regard, some issues already addressed above will be further investigated to understand the observed dynamic behaviour. These activities may include alterations of the impact hammer calibration set-up using other reference masses or sensors, the modification of the measuring chain and the data analysis procedures, comparisons of guided and manually operated hammer strokes, the analysis of phase responses and modelling using multi-degreeof-freedom systems.

\section{ACKNOWLEDGEMENT}

The authors gratefully acknowledge Ms Yang Peng for her collaboration in performing the experimental measurements presented in this paper.

\section{REFERENCES}

[1] Guideline DKD-R 3-10 Sheet 1, "Dynamic calibration of uniaxial force measuring devices and testing machines (basic principles)", June 2017. DOI: https://doi.org/10.7795/550.20190507BEN

[2] ISO 4965, "Metallic materials - Dynamic force calibration for uniaxial fatigue testing", July 2012.

[3] M. Kobusch, S. Eichstädt, "A case study in modelbased dynamic calibration of small strain gauge force transducers", Acta IMEKO 6 (2017) 1, pp. 312, DOI:

http://dx.doi.org/10.21014/acta_imeko.v6i1.433

[4] N. Vlajic, A. Chijioke, "Traceable calibration and demonstration of a portable dynamic force transfer standard", Metrologia, vol. 54, 2017, pp. 83-98, DOI: https://doi.org/10.1088/1681-7575/aa75da

[5] E. van de Bunt, J. Dekker, F. Jaouën, "Determining the dynamic behaviour of force panels for measuring wave impacts on model structures", Proc. of the 6th International Conference on Advanced Model Measurement Technology for the Maritime Industry AMT'19, Rome, Italy, 2019.

[6] Agilent, "The Fundamentals of Modal Testing", Agilent Application Note 243-3, Agilent Technologies, USA, 2000.

[7] ISO 7626-5, "Mechanical vibration and shock Experimental determination of mechanical mobility - Part 5: Measurements using impact excitation with an exciter which is not attached to the structure", 2019.

[8] M. Kobusch, L. Klaus, L. Muñiz Mendoza, "Investigation of impact hammer calibrations", in Proc. of IMEKO 23rd TC3, 13th TC5 and 4th TC22 International Conference, Helsinki, Finland, 2017. 